\title{
Exactly solvable Richardson-Gaudin models and their applications
}

\author{
S Pittel ${ }^{1}$ and J Dukelsky ${ }^{2}$ \\ ${ }^{1}$ Bartol Research Institute and Department of Physics and Astronomy, University of Delaware, \\ Newark, DE 19716, USA \\ ${ }^{2}$ Instituto de Estructura de la Materia, CSIC, Madrid, Spain \\ E-mail: pittel@bartol.udel.edu
}

Received 12 July 2005

Accepted for publication 1 September 2005

Published 28 June 2006

Online at stacks.iop.org/PhysScr/T125/91

\begin{abstract}
We first show that the quantum pairing problem can be mapped exactly on to a classical electrostatic problem in two dimensions and then use this analogy to obtain a pictorial representation of how superconductivity arises in a finite fermionic system. Specific application to the nuclei ${ }^{114-116} \mathrm{Sn}$ suggests some new insight into the evolution of pairing correlations in a quantum system with few active particles. We also summarize other recent work on exactly solvable pairing models, including their applications in a wide variety of strongly correlated quantum systems.
\end{abstract}

PACS numbers: 21.60.Cs, 21.60.Fw, 02.30.Ik

\section{Introduction}

In the early 1960s, Richardson [1] showed how to exactly solve the pure pairing model (PM) including non-degenerate single-particle orbits. Almost 40 years later, Dukelsky et al [2] showed how to generalize Richardson's solution, making use of related work by Gaudin on spin models, so that the resulting Richardson-Gaudin (R-G) models can now describe the physics of a wide variety of strongly correlated many-body quantum systems governed by pairing correlations. Many interesting applications of these exactly solvable models followed soon thereafter, to problems in nuclear physics, condensed matter physics and atomic and molecular physics. The methods have been applied both to fermion and boson systems, invariably yielding useful insight into the properties of the complex quantum systems that were modelled. A review of the R-G models and their applications can be found in [3].

More recently, several further extensions of the exactly solvable R-G models have been proposed. Along one interesting line, it has been shown how to generalize these models to describe the interplay between an atomic system (either fermionic or bosonic) that is governed by pairing correlations and another bosonic degree of freedom [4]. Such models are of great interest in the context of efforts to form molecular BEC from ultracold atomic gases and corresponding efforts to study the crossover between BEC and $\mathrm{BCS}$ in fermionic gases.

In this paper, the focus will be on one particular application of exactly solvable R-G models of relevance to nuclear structure. In particular, we will show how Richardson's exact solution of the pure PM leads to an electrostatic analogy for any quantum pairing problem and will then apply this electrostatic analogy to the problem of pairing in finite nuclei. What will emerge is a pictorial representation of how superconductivity emerges in a finite quantum system with a small number of particles, such as the atomic nucleus [5].

\section{Richardson's solution of the PM}

The pure PM Hamiltonian for fermionic systems can be written as

$$
H_{P}=\sum_{l} \epsilon_{l} \hat{N}_{l}+\frac{g}{2} \sum_{l l^{\prime}} A_{l}^{\dagger} A_{l},
$$

where

$$
\hat{N}_{l}=\sum_{m} a_{l m}^{\dagger} a_{l m}, \quad A_{l}^{\dagger}=\sum_{m} a_{l m}^{\dagger} a_{l \bar{m}}^{\dagger} .
$$

Here $a_{l m}$ creates either a boson or a fermion in singleparticle state $l m$ and $l \bar{m}$ denotes the time reverse of $l m$. 
Richardson considered the following ansatz for a system of $2 N$ particles subject to this Hamiltonian:

$$
|\Psi\rangle=\prod_{i=1}^{N} B_{\alpha}^{\dagger}|0\rangle, \quad B_{\alpha}^{\dagger}=\sum_{l} \frac{1}{2 \epsilon_{l}-e_{\alpha}} A_{l}^{\dagger} .
$$

He showed that these are indeed the exact seniority-zero (i.e., fully-paired) eigenstates of the pairing Hamiltonian if the pair energiese $_{\alpha}$ satisfy the set of equations $\left(\Omega_{l}=l+1 / 2\right)$

$$
1+2 g \sum_{l} \frac{\Omega_{l}}{2 \epsilon_{l}-e_{\alpha}}-4 g \sum_{\beta(\neq \alpha)} \frac{1}{e_{\beta}-e_{\alpha}}=0 .
$$

The coupled equations (4), one for each of the $N$ collective pairs, are called the Richardson equations. There are as many solutions to these equations as there are seniorityzero eigenstates in the Hilbert space. Corresponding to each eigenstate is an eigenenergy obtained by summing the associated pair energies, namely:

$$
E=\sum_{\alpha} e_{\alpha}
$$

Included among these solutions is the ground state, the state with lowest energy.

While the above discussion focused on the seniority-zero states, it is possible to use the same general procedure to generate all states of the system, including those with broken pairs.

\section{An electrostatic analogy for PMs}

By making use of Richardson's exact solution of the pure PM Hamiltonian, it is possible to establish an exact electrostatic analogy for the quantum pairing problem. To do so, consider the energy functional

$$
\begin{aligned}
U= & -\frac{1}{4 g}\left(\sum_{\alpha} e_{\alpha}-\sum_{j} \Omega_{j} \epsilon_{j}\right)-\frac{1}{2} \sum_{j \alpha} \Omega_{j} \ln \left|2 \epsilon_{j}-e_{\alpha}\right| \\
& -\frac{1}{2} \sum_{\alpha \neq \beta} \ln \left|e_{\alpha}-e_{\beta}\right|-\frac{1}{8} \sum_{i \neq j} \Omega_{i} \Omega_{j} \ln \left|2 \epsilon_{i}-2 \epsilon_{j}\right| .
\end{aligned}
$$

It can be readily shown that when we differentiate $U$ with respect to the pair energies $e_{\alpha}$ and equate to zero we recover precisely the Richardson equations (4).

The physical meaning of $U$ follows from the fact that the Coulomb interaction between two point charges in two dimensions is

$$
v\left(\mathbf{r}_{1}, \mathbf{r}_{2}\right)=-q_{1} q_{2} \ln \left|\mathbf{r}_{1}-\mathbf{r}_{2}\right|,
$$

where $q_{i}$ is the charge and $r_{i}$ is the position of particle $i$.

Thus, $U$ is the energy functional for a classical twodimensional (2D) electrostatic system with the following ingredients:

- There is a set of fixed charges, one for each single-particle level, which are located at the positions $2 \epsilon_{i}$ and have charges $\Omega_{i} / 2$. We will call them orbitons.
- There are $N$ free charges, one for each collective pair, which are located at the positions $e_{\alpha}$ and have positive unit charge. We will call them pairons.

- There is a Coulomb interaction between all charges.

- There is a uniform electric field in the vertical direction with intensity $1 / 4 g$.

The existence of this exact analogy suggests that we might be able to use the positions that emerge for the pairons in the classical problem to gain insight into the quantum problem, hopefully an insight that was not otherwise evident.

Some other properties of the electrostatic problem relevant to pairing in fermion systems are:

- Since the orbiton positions are given by the singleparticle energies, they lie on the vertical or real axis.

- The pair energies that emerge from the Richardson equations are not necessarily real. They can either be real or they can come in complex conjugate pairs. Thus, a pairon must either lie on the vertical axis (real pair energies) or be part of a mirror pair (complex pair energies).

\section{A new pictorial representation of nuclear superconductivity}

We now apply the electrostatic analogy to the problem of identical nucleon pairing and in particular to the question of how superconductivity arises in such systems. Because of the limited number of active nucleons in a nucleus, it is extremely difficult to see evidence for the transition to superconductivity in such systems.

We will discuss what happens when we apply the electrostatic analogy to the semi-magic nuclei ${ }^{114-116} \mathrm{Sn}$. The calculations are done as a function of pairing strength $g$, using single-particle energies extracted from experiment. Table 1 shows the corresponding information on the positions and charges of the orbitons.

Figure 1 focuses on the nucleus ${ }^{114} \mathrm{Sn}$, showing the positions of the pairons in the 2D plane as a function of $g$. Since ${ }^{114} \mathrm{Sn}$ has 14 valence neutrons, there are seven pairons in the classical picture. In the limit of very weak coupling, six neutrons fill the $d_{5 / 2}$ orbit and eight fill the $g_{7 / 2}$. The corresponding electrostatic picture (figure 1(a)) has three pairons close to the $d_{5 / 2}$ orbiton and four close to the $g_{7 / 2}$. In the figure, we draw lines connecting each pairon to the one that is closest to it. These lines make clear that at very weak coupling the pairons organize themselves as artificial atoms around their corresponding orbitons.

What happens as we increase the magnitude of $g$ (figures 1(b) and (c))? (The physical value is roughly $-0.092 \mathrm{MeV}$.) As $g$ increases, the pairons repel, causing the atoms to expand. For $g \approx-0.04$, a transition takes place from two isolated atoms to a cluster, with all pairons connected to one another. We claim that this geometrical transition from atoms to clusters in the classical problem is a reflection of the superconducting transition in the quantum problem.

We have also treated the nucleus ${ }^{116} \mathrm{Sn}$, with the same set of single-particle energies as in ${ }^{114} \mathrm{Sn}$. For weak $g$, the pairons distribute themselves into three atoms, surrounding the $\mathrm{d}_{5 / 2}, \mathrm{~g}_{7 / 2}$ and $\mathrm{s}_{1 / 2}$ orbitons. When $g$ reaches roughly 
Table 1. Position and charges of the orbitons appropriate to a pairing treatment of ${ }^{114-116} \mathrm{Sn}$.

\begin{tabular}{lll}
\hline Orbiton & Position & Charge \\
\hline $\mathrm{d}_{5 / 2}$ & 0.0 & -1.5 \\
$\mathrm{~g}_{7 / 2}$ & 0.44 & -2.0 \\
$\mathrm{~s}_{1 / 2}$ & 3.80 & -0.5 \\
$\mathrm{~d}_{3 / 2}$ & 4.40 & -1.0 \\
$\mathrm{~h}_{11 / 2}$ & 5.60 & -3.0 \\
\hline
\end{tabular}

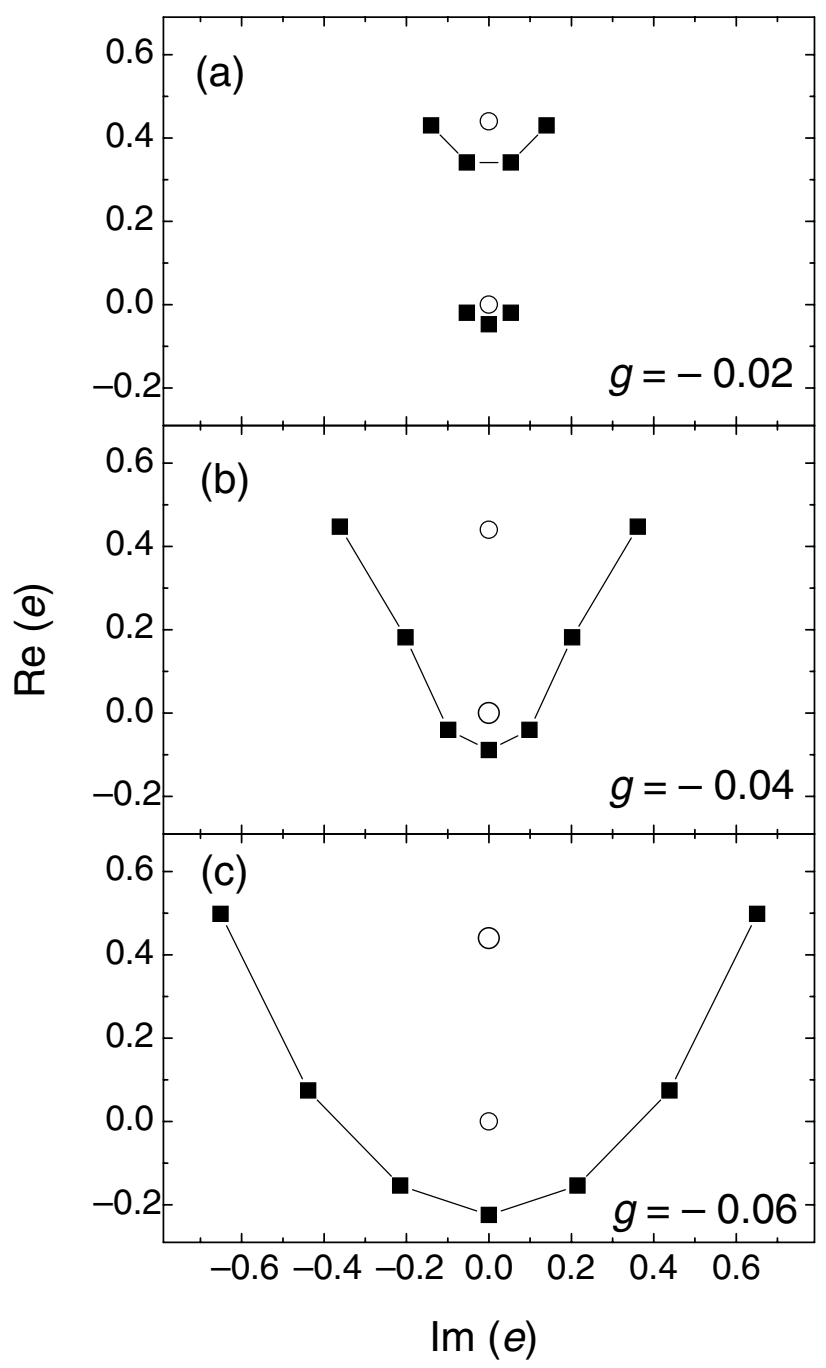

Figure 1. 2D representation of the pairon positions in ${ }^{114} \mathrm{Sn}$ for three selected values of $g$. The orbitons are represented by open circles; only the lowest two, the $\mathrm{d}_{5 / 2}$ and $\mathrm{g}_{7 / 2}$, are shown at the positions dictated by table 1 .

-0.06 , the two lowest atoms - containing seven paironsmerge into a cluster, as in ${ }^{114} \mathrm{Sn}$, with the eighth still separate. When $g$ grows to roughly -0.095 a second transition takes place, with the eighth pairon merging into a larger cluster with the other seven. From this point on, superconductivity is complete. Thus the electrostatic analogy, which emerges because of the existence of the exact Richardson solution, suggests that full superconductivity develops in stages, as the pairing interaction successively overcomes the single-particle gaps that dominate in the weak-coupling limit.

\section{Closing remarks}

In this paper, we have described how the exact solvability of the pure PM can be used to get a pictorial representation of the emergence of superconductivity in a finite fermi system that is governed by pairing correlations. Application of these ideas to the nuclei ${ }^{114-116} \mathrm{Sn}$ gave some interesting new insight into how pairing correlations evolve in a system with so few active particles.

As discussed in section 1, there have also been several other applications of exactly solvable PMs reported recently. These include applications in which the pure PM was used and others in which the pairing Hamiltonian does not have constant pairing strengths but is nevertheless exactly solvable. There have also been several new directions recently pursued. Along one line, exactly solvable PMs have been extended to include the coupling of a paired system with another bosonic degree of freedom, of possible relevance to atomic systems coupled to molecular dimers. In a slightly different context, in which the particle-hole rather than the pair realization of SU(2) is used, the same models can be used to treat multiatom systems coupled to a radiation field. Another direction under current investigation concerns the further generalization of such exactly solvable models to systems governed by other algebraic correlation structures, not just those involving SU(2) pairing. And we are optimistic that further generalization and other interesting applications are still to follow.

\section{Acknowledgments}

This work is based on a talk presented by one of the authors (SP) at the International Conference on Finite Fermionic Systems: Nilsson Model 50 Years, held in Lund, Sweden from 14-18 June 2005. The work reported herein was supported in part by the US National Science Foundation under grant no PHY-0140036 and in part by the Spanish DGI under grant no BFM2003-05316-C02-02. We also gratefully acknowledge the contributions of Carlos Esebbag to the application that was reported.

\section{References}

[1] Richardson R W 1963 Phys. Lett. 3277

Richardson R W and Sherman N 1964 Nucl. Phys. 52221

Richardson R W 1968 J. Math. Phys. 91327

[2] Dukelsky J, Esebbag C and Schuck P 2001 Phys. Rev. Lett. 87 066403

[3] Dukelsky J, Pittel S and Sierra G 2004 Rev. Mod. Phys. 76643

[4] Dukelsky J, Dussel G G, Esebbag C and Pittel S 2004 Phys. Rev. Lett. 93050403

[5] Dukelsky J, Esebbag C and Pittel S 2002 Phys. Rev. Lett. 88 062501 